\title{
Current opinions regarding care of the mature pediatric urology patient
}

\author{
K.M. Szymanski, R. Misseri, B. Whittam, T. Large and M.P. Cain
}

Division of Pediatric Urology, Riley Hospital for Children, Indiana University School of Medicine, Indianapolis, IN 46205, USA

\section{Summary}

Introduction

With continued improvements in pediatric urology care of patients with complex congenital genitourinary conditions, many survive into adulthood. This fact has created a challenging situation of transitioning from pediatric to adult care. Establishing long-term follow-up with appropriate specialists is a critical part of a successful transition to adulthood for this population.

\section{Objective}

This study sought to elucidate current practices and opinions regarding the management of adult complex genitourinary patients by pediatric urologists, in order to determine if a consensus for adult care exists.

Study design

An anonymous, 15-question online survey was created to address practice patterns and opinions regarding the transition of care of complex genitourinary patients. An invitation to participate was distributed via email to 200 pediatric urologists who were members of the American Urological Association. Complex genitourinary patients were defined broadly as those with a history of: spina

This is the author's manuscript of the article published in final edited form as:

Szymanski, K. M., Misseri, R., Whittam, B., Large, T., \& Cain, M. P. (2015). Current opinions regarding care of the mature pediatric urology patient. Journal of Pediatric Urology, 11(5), 251.e1-251.e4. 
bifida, bladder exstrophy, cloacal exstrophy, cloacal anomalies, posterior urethral valves or disorders of sex development. Fisher's exact test was used for analysis.

Results

The response rate was $31.0 \%$ (62/200). Two-thirds (67.7\%) cared for adults with complex genitourinary conditions. Overall, $51.6 \%$ of pediatric urologists felt that general urologists best follow adult patients, but only $6.5 \%$ recommended this for patients with prior complex genitourinary reconstruction ( $P$ $<0.001)$. Instead, the majority (80.6\%) felt that a pediatric or adult urologist with an interest and training in adolescent/transitional urology who routinely performs such procedures would provide optimal care. Follow-up by a primary care physician alone was not recommended. Recommendations did not change if patients had developmental delay or lived independently $(P=0.47$ and $P=0.72$, respectively). Overall, $69.4 \%$ would refer mature complex genitourinary patients to a urologist with interest and training in adolescent/transitional urology, if one was available. However, only $45.2 \%$ had such an individual available in their practice $(P<0.001)$.

Discussion

In the present study, the opinions of pediatric urologists regarding optimal providers of long-term follow-up for mature complex genitourinary patients were presented. While the results may not represent the views of the entire pediatric urology community, responses from motivated individuals with a particular interest in transition care may be especially valuable. Although the present study did not outline a mechanism for improving transitional care, it offered valuable information on prevailing opinions in this area. Finally, the opinions of mostly North American Pediatric Urologists were presented, which may not apply to other healthcare settings.

Conclusions 
Pediatric urologists appeared to be virtually unanimous in recommending that urologists provide the most appropriate long-term follow-up of patients with congenital genitourinary conditions. Specifically, $80 \%$ recommended that patients with prior complex surgical reconstruction be followed by a urologist with specific interest, training and experience in the area of transitional urology. The data suggest that this may be an unmet need of these specialists and may signify the need for specific training in the care of such patients.

\section{Introduction}

Congenital genitourinary disorders were traditionally confined to the pediatric patient population because associated spinal cord defects and complex syndromes tended to have poor survival. However, advances in medical and surgical therapy now facilitate individuals born with genitourinary disorders to live well into adulthood

. The goals of care for this complex population are similar to those of otherwise healthy adult patients under the care of general urologists. These include: preservation of renal function; prevention of upper urinary tract obstruction, infections and stones; maintenance of urinary continence; and assistance with fertility and sexual function. Achieving these goals in adolescents or adults with a history of congenital genitourinary anomalies, whether or not they have had prior reconstructive surgery, often requires significant resources, expertise and ancillary support. Currently, it appears that this process is managed by overstretched pediatric urologists and sometimes ill-prepared adult urologists . Concerns exist that a significant number of these patients are lost to urologic follow-up and subsequently receive emergent and disorganized care for the management of acute exacerbations of chronic problems. While establishing long-term follow-up with appropriate specialists is a critical part of a successful transition to 
adulthood, general consensus on who and how to best follow this population is lacking, particularly because of their heterogeneous needs and lack of consensus recommendations in the urologic literature

. The present study sought to elucidate practice and opinions regarding the management of patients with complex genitourinary conditions by practicing pediatric urologists, in order to determine if a consensus for adult care exists.

\section{Materials and methods}

A 15-question non-validated online survey was drafted to address practice patterns and opinions regarding the transition of care from adolescence to adulthood for complex pediatric urology patients. An invitation to participate was emailed to 200 pediatric urologists who were members of the American Urologic Association (AUA) (in January 2012). A further invitation was emailed 1 month later to encourage enrollment. Information was collected on practice type, years of experience and AUA section membership. Complex genitourinary conditions were broadly defined as patients with a history of: spina bifida, bladder exstrophy, cloacal exstrophy, cloacal anomalies, PUV or disorders of sex development.

The survey consisted of multiple-choice questions with free text options. A clinical scenario was used to assess comfort with the management of adult complex genitourinary patients. The scenario described a 21-year-old patient presenting with a large bladder stone and history of augmentation cystoplasty, bladder neck reconstruction and Mitrofanoff catheterizable channel. When assessing opinions regarding the most appropriate providers of long-term follow-up, whether recommendations would change if the patient had prior genitourinary reconstruction (defined as bladder augmentation, catheterizable channel or bladder neck reconstruction), developmental delay or lived independently were also examined.

The survey was administered online through Surveymonkey [ https://www.surveymonkey.com ]. Responses were analyzed using Fisher's exact test using a critical $P$-value of 0.05 . Statistical analyses were performed using Stata (v10.1) (StataCorp, USA). 


\section{Results}

Sixty-two of the 200 invited participants completed the survey, giving a $31.0 \%$ response rate. Participants originated from all eight sections of the AUA (Table 1). Responding practitioners varied in their clinical experience. The lowest percentage (22.6\%) practiced for over 20 years.

Two-thirds of pediatric urologists (67.7\%) cared for adults aged over 21 years with complex genitourinary conditions. The majority $(80.7 \%)$ felt comfortable with performing surgical procedures on older children, adolescents and young adults with complex genitourinary conditions, while $14.5 \%$ felt comfortable with only certain conditions. which particular conditions this included were not assessed. In the clinical scenario of an adult patient with prior genitourinary reconstruction and a bladder stone, $58.1 \%$ of pediatric urologists would treat the patient themselves. Others would refer the patient to another urologist with more experience or training.

When asked who should optimally follow these patients into adulthood, $51.6 \%$ felt that general urologists would be most appropriate. Another $25.8 \%$ believed that urologists (pediatric or adult) with interest and training in the management of these diseases, who also routinely perform such procedures, would provide that optimal follow-up. Another $22.6 \%$ felt that pediatric urologists should continue follow-up into adulthood. Only one participant (1.6\%) recommended long-term follow-up with a primary care physician - this was for a patient with developmental delay.

When it came to patients with prior complex reconstructive surgeries in childhood, such as bladder augmentation, catheterizable channels and bladder neck reconstructions, only $6.5 \%$ of pediatric urologists felt that general urologists would provide the most appropriate long-term follow-up. This was significantly lower than the $51.6 \%$ recommended for complex genitourinary patients overall $(P<0.001)$. An overwhelming majority of pediatric urologists $(80.6 \%)$ felt that urologists with interest and training in adolescent/transitional and who routinely performed such procedures would provide optimal follow-up. 
Another $12.9 \%$ felt that this should be a pediatric urologist. The choice of specialist for long-term followup was not affected by whether a patient had developmental delay or lived independently $(P=0.47$ and $P=0.72$, respectively).

Overall, $69.4 \%$ of pediatric urologists would refer their patients to a urologist with interest or training in adolescent/transitional care of these complex patients, if one was available in their practice. In contrast, only $45.2 \%$ had such a urologist available in their practice $(P<0.001)$, which suggests a possibly unmet need.

\section{Discussion}

Establishing appropriate long-term follow-up is a critical part of a successful transition to adulthood for pediatric patients with complex genitourinary conditions. The present study reported that while half of pediatric urologists believed that adults with complex genitourinary conditions may be best followed by general urologists, $80 \%$ recommend that it is urologists with interest, training and experience in adolescent/transitional urology who should follow patients with prior complex genitourinary reconstruction.

The fact that follow-up by a primary care physician alone was very rarely recommended underlines the view that patients with complex genitourinary conditions require life-long urological surveillance. Despite examples of transitional programs established by groups like the American College of Cardiology and the Cystic Fibrosis Foundation for patients with congenital cardiac anomalies or cystic fibrosis, similar transitional programs and standards in urology are still evolving. One transitional care model used for patients with spinal bifida involves a multidisciplinary transition clinic for adults that is similar in structure to a multidisciplinary clinic used for children and adolescents. Another model is used at the present institution: patients previously followed at the multidisciplinary spina bifida clinic are offered follow-up at a transitional urology clinic by a pediatric urologist with a particular interest in this area. A 
third model involves discharge to an adult urologist with a medical summary prepared by a pediatric urologist.

Despite some success reported by centers implementing the third model involving follow-up with an adult urologist, it is associated with several difficulties. The main difficulty is that feasibility of this transition approach hinges on an adult urologist being able and willing to take over the care of a complex genitourinary patient. This option may not exist in many communities and healthcare systems. Despite mandatory training in pediatric urology during residency, finding an adult urologist with an interest in patients with congenital urologic disease remains a challenge. Therefore, care of the complex genitourinary patient beyond childhood often falls to pediatric urologists. In addition, it was found that the vast majority of pediatric urologists believe that general urologists are not the most appropriate specialists to undertake the responsibility of this subgroup of adults with a history of prior complex genitourinary reconstruction.

Transition models that involve changing providers present several challenges. The most common problem involves poor planning. This results in a rushed and disorganized transitional process. An inadequately planned transition may contribute to patients' feelings of abandonment and decreased adherence with medical care. Having a single designated urologist for the mature complex genitourinary patient may help in transition planning. Another potential pitfall is that non-adherence and loss to follow-up may be related to poorly expressed expectations on the part of the patient or the expectations not being met by the accepting urologist, particularly regarding sexual health . Understandably, many pediatric and adult urologists do not have interest, knowledge or training in these emerging areas of adult care. It would be in the patient's best interest if the accepting urologists had both the motivation and experience to address their concern. Furthermore, successful transition programs are often time consuming and inadequate payment may make caring for such patients 
financially burdensome for many practitioners. Additional efforts are necessary to improve payment for transition services and to ensure that patients have insurance coverage.

Indeed, there appears to be a paucity of appropriate specialists in the field of transitional urology. Although $70 \%$ of pediatric urologists felt they would refer their patients to a urologist specializing in transitional care, less than half actually had a such a colleague in their practice. Meeting the increasing needs of a growing population of adults with complex genitourinary conditions will likely require practice and workforce changes. According to the American Board of Urology, the care of adults by a urologist with a subspecialty certification in Pediatric Urology may not exceed $25 \%$ of their practice. One possible solution would involve establishing a separate urologic subspecialty to provide a seamless transition through adolescence into adulthood. Such subspecialists would likely benefit from advanced training in pediatric urology and reconstructive urology. The goal for such a subspecialty would be to provide expert multifaceted care to adult complex genitourinary patients within a network of providers and resources to encourage patient adherence and improve long-term outcomes. At a minimum, resident and fellowship training should involve more education on transitional care of the pediatric genitourinary patient.

In a time, when preventative care models are being implemented to reduce healthcare costs, transitional care for the complex genitourinary patient should be a primary goal for this specialty. Without routine monitoring, patients will likely present requiring major emergent interventions because of a relative paucity of symptoms associated with degenerative changes of the genitourinary system. This may be further exacerbated as the adolescent and young adult may have poor self-management skills, poor understanding of their disease and decreased parental supervision, while overall assistance may diminish or disappear as the caregiver ages or dies. Ultimately, a 'congenitalist' offers the potential for marked cost reduction within this complex patient population through routine follow-up 
appointments and small interventions rather than extensive and costly surgery after extended periods of loss to follow-up.

Transitioning to a qualified adult urology care may also apply to patients with less complex genitourinary conditions, such as girls with refractory overactive bladder or boys with difficult to treat balanitis xerotica obliterans. In addition, the present study focused on urological transition, but a comprehensive transition to adult care may need to incorporate several different subspecialties. Depending on the patient, these services may include nephrology, colorectal surgery, gynecology, neurosurgery, orthopedics or physiotherapy.

The present study had several limitations. The results may not represent the views of the entire pediatric urology community. On the other hand, responses from pediatric urologists who were particularly motivated and interested in transition care may be especially valuable in this new area of care. The response rate was modest, but comparable to other clinical surveys. In addition, general urologists, who often take over care of complex genitourinary patients, were not included in the study. This will be an area of future investigation. Although this study did not outline a mechanism for improving the transitional process, it is felt that it offered valuable information on trends in opinion regarding transitional care. Finally, the opinions of mostly North American pediatric urologists were presented; these may not apply to other healthcare settings, whether due to resource availability or training. While North American fellowship-trained pediatric urologists typically have little exposure to pediatric surgery after a rotation during residency training, this may be different in European or other healthcare systems.

\section{Conclusions}

Pediatric urologists almost unanimously agree that a urologist is best suited to provide the long-term urologic follow-up of patients with congenital genitourinary conditions. Specifically, the majority 
recommends that patients with prior complex genitourinary reconstruction as a child should be followed by a urologist with specific interest, training and experience in the area of transitional urology. The data suggest there may be an unmet need in this area.

\section{Conflicts of interest}

None.

Research support

None.

Internal Review Board approval

Exempt. 


\section{References}

[1]. Wood D.: Adolescent urology: developing lifelong care for congenital anomalies. Nat Rev Urol 2014; 11: pp. 289-296

[2]. Tanaka S.T., Kaufman M.R., and Brock J.W.: The aging pediatric urology patient: obstacles and opportunities in transition care. J Urol 2012; 187: pp. 1159-1160

[3]. Woodhouse C.R., Neild G.H., Yu R.N., and Bauer S.: Adult care of children from pediatric urology. J Urol 2012; 187: pp. 1164-1171

[4]. Misseri R.: Dialogues in pediatric urology transition to adulthood: concerns and considerations for the pediatric urologist. Dialogues Pediatr Urol 2013; 34: pp. 3

[5]. Summers S.J., Elliott S., McAdams S., Oottamasathien S., Brant W.O., Presson A.P., et al: Urologic problems in spina bifida patients transitioning to adult care. Urology 2014; 84: pp. 440-444

[6]. Woodhouse C.R., Lipshultz L., Hwang K., Mouriquand P., and Creighton S.: Adult care of children from pediatric urology: part 2. J Urol 2012; 188: pp. 717-723

[7]. Cooley W.C., and Sagerman P.J.: Supporting the health care transition from adolescence to adulthood in the medical home. Pediatrics 2011; 128: pp. 182-200

[8]. Sawin K.J., Bellin M.H., Roux G., Buran C.F., and Brei T.J.: The experience of self-management in adolescent women with spina bifida. Rehabil Nurs 2009; 34: pp. 26-38

[9]. Woodhouse C.R.: Myelomeningocele in young adults. BJU Int 2005; 95: pp. 223-23 
Table 1. Participant characteristics.

\begin{tabular}{|ll|}
\hline \hline \multicolumn{1}{|c|}{ Characteristic } & \multicolumn{1}{c|}{ Number (\%) } \\
\hline \hline \multicolumn{2}{|c|}{ American Urological } \\
\hline Northeastern & $7(11.3 \%)$ \\
\hline New England & $5(8.1 \%)$ \\
\hline New York & $1(1.6 \%)$ \\
\hline Mid-Atlantic & $2(3.2 \%)$ \\
\hline \hline Southeastern & $7(11.3 \%)$ \\
\hline North Central & $13(21.0 \%)$ \\
\hline Western & $14(22.6 \%)$ \\
\hline \hline South Central & $8(12.9 \%)$ \\
\hline Outside United States & $5(8.1 \%)$ \\
\hline Years in practice & \\
\hline Less than 10 years & $27(43.5 \%)$ \\
\hline $11-20$ years & $20(32.3 \%)$ \\
\hline \hline Over 20 years & $14(22.6 \%)$ \\
\hline Unknown & $1(1.6 \%)$ \\
\hline \hline
\end{tabular}

